\title{
WHAT DETERMINES THE SITE OF INFLAMMATION IN UVEITIS AND CHORIORETINITIS?
}

\author{
JOHN V. FORRESTER ${ }^{1}$, JANET LIVERSIDGE ${ }^{1}$, ANDREW DICK ${ }^{1}$, PAUL MCMENAMIN $^{2}$, \\ MARIA KUPPNER ${ }^{1}$, ISOBEL CRANE ${ }^{1}$ and PARWEZ HOSSAIN ${ }^{1}$ \\ Aberdeen
}

Uveitis is a relatively confusing term since it implies primary inflammation of the uveal tract. However, it has become clear during the last few decades of research into the causes of uveitis that the uveal tract acts primarily as the conduit for the inflammatory cells and the increased blood flow that accompany inflammation targeted to other sites in the eye such as the retina and sclera. Uveitis is, therefore, better referred to as intraocular inflammation (IOI).

A major problem in understanding the pathophysiology of IOI is that clinically there is a great variety of presentations of apparently different conditions. However, a significant unifying concept, at least from the viewpoint of pathogenetic mechanisms, is that many of the clinical manifestations of IOI can be mimicked with models of experimental autoimmune uveoretinitis (EAU) which utilise a single antigen. ${ }^{1}$ This is not to say that autoimmune mechanisms account for most causes of IOI since the opposite is probably true; rather, that the intraocular tissues, particularly the retina and choroid, can respond to challenge by foreign or autoantigen in only a limited set of ways and that it should not be surprising that there is considerable overlap in the clinical manifestations of many of these conditions. These concepts have been discussed previously. ${ }^{2.3}$

This paper aims to address a separate but related problem: if the eye and its component tissues can respond in only a limited set of clinical manifestations, why is there preferential localisation of inflammation and tissue damage to discrete sites in certain conditions?

From: ${ }^{1}$ Department of Ophthalmology, University of Aberdeen, Aberdeen, UK; ${ }^{2}$ Department of Anatomy and Human Biology, University of Western Australia.

Correspondence to: Professor J. V. Forrester, Department of Ophthalmology, University of Aberdeen, Medical School, Foresterhill, Aberdeen AB9 2ZD, UK.

\section{CLASSIFICATION OF INTRAOCULAR INFLAMMATION}

In essence, the answer to this question lies in the cause of the IOI. For instance, cytomegalovirus (CMV) retinitis with choroiditis is clearly localised to the retina and pars planitis is localised to the pars plana. However, further analysis leads to blurring of these distinctions in many of the conditions. Pars planitis is a subset of what is termed intermediate uveitis' according to the International Uveitis Study Group Classification ${ }^{4}$ and intermediate uveitis is caused by or associated with several conditions including multiple sclerosis and sarcoidosis. However, sarcoidosis can also cause retinitis and/or choroiditis in isolation, so what constitutes sarcoidosis-related IOI? Furthermore, pars planitis is essentially a clinical diagnosis which can be confused, albeit rarely, with other conditions such as Toxocara uveitis.

Accordingly, while it is important to make specific diagnoses where possible, it is also necessary to retain a pathogenetic view of the inflammatory condition and to consider that any particular presentation of IOI represents a stage in the spectrum of clinical severity that may accompany any form of IOI.

In order to keep this perspective, a simple classification of IOI is required. IOI may therefore be categorised according to site as anterior or posterior, and according to cause as infective or non-infective.

Anterior IOI refers to all intraocular inflammatory conditions in which the primary site of inflammation is located anterior to the iris-lens diaphragm and essentially represents all types of iritis and iridocyclitis; posterior IOI refers to all intraocular inflammatory conditions occurring posterior to the iris-lens diaphragm and includes pars planitis, intermediate uveitis, vitritis, all forms of chorioretinitis and 
choroiditis, retinitis and retinal vasculitis. This classification has been described before and relates to the general pathogenetic mechanisms involved in each category of IOI. ${ }^{2}$ Keratitis and scleritis are not normally included in this classification, even if they are associated with secondary uveitis. ${ }^{5}$

Anterior IOI may be acute recurrent or chronic and direct infective causes are uncommon. In noninfective anterior uveitis MHC class I mediated mechanisms appear to be important since approximately $50 \%$ of cases of acute anterior uveitis are linked to HLA-B27 and its associated conditions. ${ }^{6}$ Interestingly, a tenuous link to Gram-negative bacteria in both the ocular and non-ocular HLAB27 disease has been suggested by several groups but not firmly established. ${ }^{7-9}$

Posterior IOI takes many forms some of which are clearly infective, such as Toxoplasma chorioretinitis and herpes simplex (HSV) retinitis, while others are apparently non-infective such as idiopathic retinal vasculitis, and sympathetic ophthalmia. Some noninfective conditions may be part of a wider systemic disorder such as Behçet's disease or multiple sclerosis, but this may apply equally to infective conditions such as leprosy, onchocerciasis and miliary tuberculosis. Others may be considered infective in certain circumstances, e.g. presumed ocular histoplasmosis, but have striking phenotypic similarity to non-infective causes of multifocal choroiditis with subretinal neovascular membrane formation. ${ }^{10}$ On the basis of their clinical and (immuno)histological resemblance to models of EAU, many of these conditions are assumed to be MHC class II mediated. ${ }^{1}$ However, in some cases MHC class I mechanisms may be important also, such as in birdshot retinochoroidopathy (HLA A29) $)^{11,12}$ and Behçet's disease (HLA B51). ${ }^{13}$

On this basis, several factors can be suggested which are likely to affect the site of primary inflammation within the eye. These include the nature and localisation of the antigen; the degree of systemic immune cell activation; access of activated immune cells to the antigenic site; the potential for local antigen presentation; and the influence of the local microenvironment in which the inflammation takes place.

\section{NATURE AND LOCALISATION OF THE ANTIGEN}

The nature and localisation of the antigen are paramount in determining the site of inflammation. For instance, the Toxoplasma parasite has a predilection for neural tissue, especially the eye and the brain (for review see Pavesio et al. ${ }^{14}$ ). However, in the retina it can persist in the encysted state without causing significant inflammation. Experimentally, this has also been found to hold true and it appears that a retinal inflammation only develops when the cysts rupture. In contrast, it is rare to find this organism in the choroid or indeed at any site in the uveal tract, and if present it sets up a marked inflammation.

Several viral organisms also have a clear neurotropism, such as HSV and CMV, while mycobacteria primarily locate in the uveal tract or optic nerve. The location of autoantigens in the photoreceptors ${ }^{15}$ or in the iris pigment epithelium ${ }^{16}$ clearly determines the site of inflammation, characterised for instance by the Dalen-Fuchs' nodule in sympathetic ophthal$\mathrm{mia}^{17}$ (see below). Conditions such as serpiginous choroiditis are clearly located to the retinal pigment epithelium (RPE), but it is not known whether the antigen is an RPE-specific autoantigen or a foreign antigen in virus-infected RPE cells. However, some ophthalmologists have treated this condition empirically with anti-viral agents on the basis that HSV may be the infective agent.

Each of these antigens will have a different effect based, in the case of foreign antigens, on its virulence and strain, and, in the case of autoantigens, determined by its immunogenicity.

\section{THE DEGREE OF SYSTEMIC IMMUNE CELL ACTIVATION}

Uveitis or IOI can be regarded as a systemic illness in which the eye is the target tissue, in much the same way as many arthritides or other organ-specific diseases are, such as thyroiditis. This concept fits well with many infective causes of uveitis in which an initial exposure to antigen such as Toxoplasma or Epstein-Barr (EB) virus leads to a prodromal 'flulike illness involving mild fever and lymphadenopathy. This leads to an acquired immune response which after initial resolution of the illness leaves a pool of antigen-specific memory $\mathrm{T}$ cells circulating through the lymphoid tissues. If the original antigen is not completely removed but finds its way to a sequestered site in the tissues, it may induce recurrent attacks of inflammation even when the memory $\mathrm{T}$ cells are non-specifically activated, e.g. on exposure to cytokines such as interferon-gamma $(\mathrm{IFN} \gamma)$ and tumour necrosis factor alpha (TNF $\alpha)$ released during the course of an intercurrent infection from an unrelated virus. Ophthalmologists have long recognised the association between minor febrile illness and the reactivation of uveitis.

In a similar way apparently unrelated infections may cause reactivation of autoimmune disease through postulated mechanisms such as molecular mimicry. According to this notion, homology between autoantigens and foreign antigens ensures that during activation of $\mathrm{T}$ cells occurring in response to an infection, $T$ cells specific for the autoantigen as well as for the foreign antigen are primed due to 
presentation of autoantigen 'look-a-like' peptides derived from the micro-organism. Such primed $T$ cells, if they find their target antigen in the tissue, can then be activated locally by appropriate antigen presenting cells. ${ }^{3}$ Thus, autoimmune disease induced by this mechanism is initiated in the periphery as part of a systemic inflammatory response. It can also be seen how the degree of $\mathrm{T}$ cell priming will determine in part whether an attack of IOI will occur.

\section{ACCESS OF PRIMED IMMUNE CELLS TO ANTIGENIC SITES}

Access to tissues by circulating lymphoid cells is restricted by tissue barriers. In the central nervous system (CNS) the blood-CNS barrier is highly developed and under normal circumstances is an effective barrier to cells and molecules. In the eye, the blood-retinal barrier is equally efficient even though it is distributed at two sites, i.e. the retinal vasculature and the RPE. In the uveal tract there is effectively no barrier to proteins, with ready exchange of molecules between the tissue space and the vessels. There may be some degree of restriction to the movement of cells which depends on the expression of adhesion molecules for transendothelial migration. However, several adhesion molecules and integrin receptors (see below) are expressed constitutively in the choroid ${ }^{18}$ suggesting that extravasation of cells in the choroid may readily occur.

The blood-ocular barrier at the ciliary body is also reasonably effective, being formed in this case by the double layer of epithelial cells, and inhibiting transfer of cells and molecules from the fenestrated ciliary body vessels. However, the barrier at the iris is less effective: posterior movement of cells and molecules is prevented by the posterior iris epithelium, which is continuous with the ciliary body epithelium, but anteriorly there is no effective barrier between the iris stroma and the anterior chamber. However, under normal circumstances there is little transport of cells and molecules at the iris vessels unless there is significant engorgement as in inflammation.

In fact, it has recently become clear that the iris vessels are the first to be breached by cells in models of EAU. ${ }^{19}$ This is followed within hours by cellular efflux from the ciliary body vessels and vessels in the choroid. Retinal vessels and the RPE layer are the last to be breached in EAU despite the fact that the photoreceptor is the target cell. ${ }^{20}$

In real terms, of course, breakdown of the blood-retinal barrier occurs during the early stages of inflammation at all sites including the bloodretinal barrier. Indeed, prior to the extravasation of cells from the retinal vessels there are morphological and immunological changes which present as 'highendothelial venule'-like changes and expression of adhesion and activation molecules, such as MHC class II and intracellular cell adhesion molecule 1 (ICAM-1). ${ }^{20}$ Such changes are probably induced through systemic cytokine activation, especially IFN $\gamma$, interleukin-1 (IL-1) and TNF $\alpha$ derived from primed $\mathrm{T}$ cells and monocytes. ${ }^{21-23}$ This synergistic interaction between the $\mathrm{T}$ cell and the target tissue is central to the development of the inflammation.

It can be predicted, therefore, that the site of inflammation will in part be determined by changes in the vessels peculiar to that tissue. There is now considerable evidence that the microvasculature of each tissue has specific properties, a little like a postal code, for targeting cells to that tissue, and thus conditions such as pars planitis and idiopathic retinal vasculitis occur because the vessels in those tissues are expressing the appropriate markers while the vessels in other tissue have remained quiescent. How this mechanism is achieved is not clear. However, there is potential for manipulating this in therapeutic terms since blocking the expression of adhesion molecules with specific antibodies and/or analogous peptides is an effective mechanism for downregulating and/or preventing inflammation.

\section{LOCAL ANTIGEN PRESENTATION AT THE TARGET SITE}

With most of the players in place at the target site, i.e. the antigen, the primed $\mathrm{T}$ cells and potential effector monocytes/macrophages, and the activated, leaky blood-tissue barrier, there is an essential requirement for cells which can present the antigen to the $\mathrm{T}$ cells and thus allow activation of the effector cells. In the eye, it was previously assumed that part of the condition of immunological privilege was due to a lack of 'professional' antigen presenting cells (APCs). ${ }^{24-26}$ (a second component being a lack of tissue lymphatics). However, it is now clear that there are rich networks of dendritic cells and resident macrophages ${ }^{27}$ within the uveal tract although there are no APCs in the retina proper ${ }^{28}$ (it has also been shown recently that there are lymphatic communications between the intraocular and the upper cervical lymph nodes. ${ }^{29}$ Dendritic cells in the choroid lie in close apposition to the RPE, i.e. at the site of the blood-retinal barrier, and are likely to be inducers of the microgranulomas found in many uveitis conditions such as sympathetic ophthalmia, multifocal choroiditis and the numerous white dot syndromes. ${ }^{17}$ In fact their distribution may explain the clinical presentation of these disorders. ${ }^{17}$

Similarly, dendritic cells in the iris probably underlie the pathology of iris granulomas and their numbers in the ciliary body may account for the frequency of cyclitis in many IOI syndromes. Remarkably, the turnover of dendritic cells in the eye is only a few days, compared with the longer 
turnover time of their counterpart in the skin, the Langerhans cells. $^{30}$

In vitro studies have shown that ocular dendritic cells have the capacity to present retinal antigens to naive and sensitised $T$ cells and are much more potent in their antigen presenting ability than macrophages. ${ }^{31}$ Resident macrophages, however, do play an accessory, supportive role in this process (Forrester, in preparation).

In the retina itself, dendritic cells do not occur. Instead the resident tissue macrophage is the microglial cell, and it has previously been suggested that this cell may act as the local APC within the retina. However, recent studies have shown that microglial cells are in fact poor presenters of antigen and may even have immunosuppressive properties themselves.

Since most inflammatory responses in CNS neural tissue commence around blood vessels as a perivascular cuff, perivascular macrophages have been implicated in local antigen presentation. These rare cells also occur in the retina and currently represent the likeliest candidate as the professional APC.

\section{REGULATION OF INTRAOCULAR INFLAMMATION BY THE LOCAL MICROENVIRONMENT}

From the foregoing discussion it might appear that IOI should occur with much greater frequency than it does. That it does not is due to mechanisms which exist in the eye to limit the inflammation and the resulting damage. This has been recognised for very many years and has been the subject of numerous immunological investigations.

In the anterior chamber ocular immune privilege has been linked to a phenomenon known as ACAID (anterior-chamber-associated immune deviation) in which it has been shown that antigens introduced into the eye not only fail to elicit a recognised immune response, but also suppress the immune response in other tissue such as the skin when these tissues are subsequently challenged with the same antigen. The effect is antigen specific and requires an intact spleen. How ACAID is induced is the subject of intense research and has been attributed to several mediators such as transforming growth factor beta (TGF $\beta$ ) and vasoactive intestinal polypeptide (VIP). ${ }^{24}$ In addition, it has recently been suggested that the constitutive expression of the apoptosisinducing molecule FasL on ocular tissues may also have a role in the process of immune privilege. ${ }^{32}$

In the posterior segment, resident tissue cells also have immunosuppressive properties. For instance, Müller cells are known to inhibit activation and proliferation of antigen-specific T cells. ${ }^{33}$ In addition, the RPE cell has both pro- and anti-proliferative effects on $\mathrm{T}$ cells. These effects are particularly regulated by the cytokines to which the $T$ cells are exposed. Under normal conditions, the RPE cell produces inhibitory mediators such as prostaglandin $\mathrm{E}_{2}\left(\mathrm{PGE}_{2}\right)$ and nitric oxide (NO) and in general deactivates dendritic cell function. However, if exposed to pro-inflammatory cytokines such as IL1 , the RPE cell can be induced to secrete cytokines and chemokines which attract $\mathrm{T}$ cells and macrophages and generate an immune response. In contrast, if exposed to TGF $\beta$ these cells can instead release anti-inflammatory cytokines such as IL-6 which shunt the immune response towards antibody production and away from tissue damage. These mechanisms have been reviewed elsewhere. ${ }^{34}$

\section{CONCLUSION}

In conclusion, therefore, it can be seen that there are several factors which determine the site at which IOI will be initiated if not restricted.

For foreign antigens, these include the natural predilection of micro-organisms for certain cells and tissues, the systemic activation of memory $\mathrm{T}$ cells in recurrent infections, the release and/or expression of antigen locally within the tissues and the ease with which local antigen presentation can overwhelm the tissue inhibitory effects.

For self antigens, the same general factors also hold true. In addition, there must be mechanisms acting to break self-tolerance within the tissue which depend somewhat on the tissue specificity of the antigen. For instance, S-antigen-like molecules can be found in other tissues but IRBP is restricted to the photoreceptor matrix.

This work was supported by the Guide Dogs for the Blind, the Leverhulme Trust and the Wellcome Trust.

\section{REFERENCES}

1. Forrester JV, Liversidge JM, Dua HS, Towler HM, McMenamin PG. Comparison of clinical and experimental uveitis. Curr Eye Res 1990;9(Suppl):75-84.

2. Forrester JV. Uveitis: pathogenesis. Lancet 1991;38: $1498-501$.

3. Forrester JV. Duke Elder lecture. New concepts on the role of autoimmunity in the pathogenesis of uveitis. Eye 1992;6:433.

4. Bloch-Michel E, Nussenblatt RB. International Uveitis Study Group recommendations for the evaluation of intra-ocular inflammatory disease. Am J Ophthalmol 1987;103:234-5.

5. Forrester JV. Uveitis. Br J Ophthalmol 1990.

6. Feltkamp TEW. Ophthalmological significance of HLA associated uveitis. Eye 1990;4:839-44.

7. Wakefield D, Stahlberg TH, Toivanen A, Ganfors K, Tennant C. Serologic evidence of Yersinia infections in patients with anterior uveitis. Arch Ophthalmol 1990;108:219-21.

8. Feltkamp TEW. Are gram negative bacteria involved in HLA-B27 associated uveitis? $\mathrm{Br} \mathrm{J}$ Ophthalmol 1995;79:718.

9. Feltkamp TEW. The pathogenetic role of HLA-B27. Clin Rheumatol 1996;15(Suppl). 
10. Saraux H, Pelosse B, Guigui A. Multifocal inner choroiditis: pseudohistoplasmosis - the European form of presumed American histoplasmosis. J Fr Ophtalmol 1986;9:645-51.

11. Nussenblatt RB, Mittal KK, Ryan SJ, Green WR, Maumenee AE. Birdshot retinochoroidopathy associated with HLA-A29 antigen and immune responsiveness to retinal S-antigen. Am J Ophthalmol 1982;94:147-56.

12. Priem HA, Kijlstra A, Noens L, Baarsma GS, DeLaey $\mathrm{J}$-J, Oosterhuis JA. HLA typing in birdshot chorioretinopathy. Am J Ophthalmol 1988;105:182-6.

13. Chajek-Shaul T, Pisanty S, Knobler H. HLA-B51 may serve as an immunogenetic marker for a subset for a subgroup of patients with Behçet's disease. Am J Med 1987;83:666-76.

14. Pavesio CE, Lightman SL, Nichols BA. Toxoplasma gondii and ocular toxoplasmosis: pathogenesis. $\mathrm{Br} \mathbf{J}$ Ophthalmol 1996; in press.

15. Gery I. Retinal antigens and the immunopathologic process they provoke. Prog Retinal Res 1986;5:75-109.

16. Broekhuyse RM, Kuhlmann ED, Winkens HJ. Experimental autoimmune anterior uveitis (EAAU). III. Induction by immunisation with purified uveal and skin melanins. Exp Eye Res 1993;56:401-11.

17. Ben Ezra D, Forester JV. White dot syndrome. Br J Ophthalmol 1995.

18. Kuppner MC, Liversidge J, McKillop-Smith S, Lumsden L, Forrester JV. Adhesion molecule expression in acute and fibrotic sympathetic ophthalmia. Curr Eye Res 1993;10:923-34.

19. Butler TL, McMenamin PG. Resident and infiltrating immune cells in the uveal tract in the early and late stages of experimental autoimmune uveoretinitis. Invest Ophthalmol Vis Sci 1996;37:2195-210.

20. McMenamin PG, Broekhuyse RM, Forrester JV. Ultrastructural pathology of experimental autoimmune uveitis: a review. Micron 1992;24:521-46.

21. Liversidge JM, Sewell HF, Forrester JV. Human retinal pigment epithelial cells differentially express MHC class II (HLA DP, DR and DQ) antigens in response to in vitro stimulation with lymphokine or purified IFN-gamma. Clin Exp Immunol 1988;73:489-94.

22. Liversidge JM, Sewell HF, Forrester JV. Interactions between lymphocytes and cells of the blood-retina barrier: mechanisms of $\mathrm{T}$ lymphocyte adhesion to human retinal capillary endothelial cells and retinal pigment epithelial cells in vitro. Immunology 1990;71:390-6.

23. Liversidge JL, Forrester JV. Antigen processing and presentation in the eye: a review. Curr Eye Res 1992;11(Suppl):49-58.

24. Streilein JW, Willbanks GA, Cousins SW. Immunoregulatory mechanisms of the eye. J Neuroimmunol 1992;39:185-200.

25. Streilein JW. Immune regulation and the eye: a dangerous compromise. FASEB J 1987;75:199-208.

26. Willbanks GA, Streilein JW. Fluids from immune privileged sites endow macrophages with the capacity to induce antigen-specific immune deviation via a mechanism involving transforming growth factor beta. Eur J Immunol 1992;22:165-74.

27. McMenamin PG, Crewe J, Morrison S, Holt PG. Immunomorphological studies of macrophages and MHC class II-positive dendritic cells in the iris and ciliary body of the rat, mouse and human eye. Invest Ophthalmol Vis Sci 1994;35:3234-50.

28. Forrester JV, McMenamin PG, Holthouse I, Lumsden L, Liversidge J. Localisation and characterisation of major histocompatibility complex class II-positive cells in the posterior segment of the eye: implications for the induction of autoimmune uveoretinitis. Invest Ophthalmol Vis Sci 1994;35:64-77.

29. Egan RM, Yorkey C, Black R, Loh WK, Stevens JL, Woodward JG. Peptide-specific T cell clonal expansion in vivo following immunization in the eye, an immune privileged site. J Immunol 1995; 157:2262-71.

30. Steptoe R. Phenotypic analysis of iris dendritic cells and macrophages. Immunology 1996;in press.

31. Steptoe R, Holt PG, McMenamin PG. Demonstration of the immunostimulatory capacity of dendritic cells isolated from the rat iris. Immunology 1995;85:630-7.

32. Griffith TS, Brunner T, Fletcher SM, Green DR, Ferguson TA. Fas ligand-induced apoptosis as a mechanism of immune privilege. Science 1995; 270:1189-92.

33. Roberge FG, Caspi RR, Nussenblatt RB. Glial retinal Müller cells produce IL-1 activity and have a dual effect on autoimmune $\mathrm{T}$ helper lymphocytes. J Immunol 1988;140:2193-6.

34. Forrester JV, Lumsden L, Liversidge J, Kuppner M, Mesri M. Immunoregulation of uveoretinal inflammation. Prog Retinal Eye Res 1995;14:393-412. 